\title{
Didáctica y lingüística: un desafío desde la universidad para la educación básica*
}

\author{
MIREYA CISNEROS ESTUPIÑÁN \\ mireyace@gmail.com \\ GIOHANNY OLAVE ARIAS ${ }^{* * *}$ \\ olavearias@gmail.com \\ ILENE ROJAS GARCÍA***** \\ zellene7@gmail.com
}

Recepción: 18 de febrero de 2015

Aprobación: 10 de mayo de 2015

Forma de citar este artículo: Cisneros Estupiñán, M., Olave Arias, G., \& Rojas García, I. (2015). Didáctica y Linguiística: un desafío desde la universidad para la educación básica. Cuadernos de Lingüística Hispánica, 26, 159-174, Tunja: Uptc.

\footnotetext{
* $\quad$ Este artículo de investigación es derivado del proyecto Enseñar a enseñar lingüística escolar: de la formación universitaria a la experiencia profesoral en didáctica de la lengua materna, inscrito en la Vicerrectoría de Investigación, innovación y extensión de la Universidad Tecnológica de Pereira, Colombia.

** Profesora Titular de la Universidad Tecnológica de Pereira, Investigadora Senior de Colciencias.

*** Doctorando de la Universidad de Buenos Aires. Investigador del CONICET Argentina.

****** Doctoranda de la Universidad del Norte, Barranquilla, Colombia.
} 


\title{
Resumen
}

Aunque se ha avanzado considerablemente en las orientaciones sobre la enseñanza de la lengua materna, persiste una escasez de estudios orientados, no solo al tema de la lectura y la escritura, sino a una didáctica más global que incluya la linguística como objeto de saber, base para el desarrollo de la competencia comunicativa. Tal situación repercute en los procesos de formación de los programas de Licenciatura en Español y Literatura donde se enfatiza en la enseñanza de la teoría lingüística, más que en la búsqueda de alternativas didácticas adaptables a las necesidades del contexto y a los objetivos de enseñanza. El presente artículo reflexiona sobre la adecuación de los saberes impartidos en la formación de docentes de Español, en el marco de una transposición didáctica entre la linguística teórica y la linguiística escolar.

Palabras clave: didáctica de la lengua materna, formación docente, lectura y escritura, transposición didáctica.

\section{Didactics and linguistics: a challenge from university to basic education.}

\begin{abstract}
There have been considerable advancements in the guidelines of mother language teaching, nevertheless, a lack of studies persists, related both to the topic of reading and writing and to a more global didactics which includes linguistics as an object of knowledge and a basis for the development of the communicative competence. This situation has consequences in the processes of education of the Licenciatura en Español y Literatura program, in which a wider emphasis is made on teaching linguistic theory than on inquiry into didactic alternatives adaptable to the necessities of the context and the objectives of teaching. This article reflects on the adaptation of knowledge included in the training of Spanish teachers, seeking to enable a didactic transposition between theoretical linguistics and school linguistics.
\end{abstract}

Key words: Mother Tongue Didactics, teacher training, reading and writing, didactic transposition. 


\section{Didactique et linguistique: un défi dans l'université pour l'éducation de base}

\section{Résumé}

Bien que l'on ait considérablement avancé dans les orientations sur l'enseignement de la langue maternelle, il persiste un manque d'études orientées, non seulement aux sujets de la lecture et de l'écriture, mais à une didactique plus globale qui inclue la linguistique en tant que savoir, base pour le développement de la compétence communicative. Une telle situation répercute aux processus de formation du programme de la Licence en Espagnol et de celui de Littérature, où l'on met l'accent sur l'enseignement de la théorie linguistique, plus que sur la recherche des alternatives didactiques adaptables aux besoins du contexte et aux objectifs de l'enseignement. Cet article propose une réflexion sur l'adéquation des savoirs impartis dans la formation des enseignants d'espagnol, dans le cadre d'une transposition didactique entre la linguistique théorique et la linguistique scolaire.

Mots clés: didactique de la langue maternelle, formation des enseignants, lecture et écriture, transposition didactique.

\section{Didática e Linguística: um desafio desde a universidade para a educação básica}

\section{Resumo}

Ainda que se tenha avançado consideravelmente nas orientações sobre o ensino da língua materna, persiste uma escassez de estudos orientados, não somente ao tema da leitura e da escritura, senão a uma didática mais global que inclua a linguística como objeto de saber, base para o desenvolvimento da competência comunicativa. Tal situação repercute nos processos de formação dos programas de Licenciatura em Espanhol e Literatura onde se enfatiza no ensino da teoria linguística mais do que na busca de alternativas didáticas adaptáveis às necessidades do contexto e aos objetivos de ensino. 0 presente artigo reflete sobre a adequação dos saberes ministrados na formação de docentes de Espanhol, no marco de uma transposição didática entre a linguística teórica e a linguística escolar.

Palavras chave: didática da língua materna, formação docente, leitura e escritura, transposição didática. 


\section{Introducción}

Este artículo da cuenta de un proyecto de investigación que se desarrolla en la Universidad Tecnológica de Pereira, el cual considera que la formación de docentes de lengua materna en la Universidad ha enfrentado dificultades al vincular la lingüística teórica con la formación en contextos reales de comunicación, dentro y fuera de la escuela.

Se trata de un desafío abordado al mismo tiempo por la didáctica y por la lingüística aplicada, pero generalmente de una manera aislada, enfatizando en lo teórico con detrimento de lo empírico, o bien, en lo técnico con detrimento de lo reflexivo, y con resultados que transparentan la ausencia de un diálogo interdisciplinar cuya urgencia radica en la necesidad de trascender la profesionalización de los licenciados en lengua materna, de la reproducción de prácticas formativas a la capacidad de proponerlas pertinentemente, de acuerdo con lo que demandan los estudiantes como sujetos sociales situados en sus entornos comunicativos particulares.

Se busca, como parte de los resultados de este proyecto, proponer estrategias de transposición didáctica de la lingüística teórica a una lingüística escolar, para integrarlas a los currículos de las licenciaturas relacionadas con la lengua materna. Dicho objetivo se alcanzará a través de una metodología cualitativa que aborde, en una primera fase, la descripción del objeto problemático a través de la hermenéutica documental y las entrevistas narrativas a los docentes en ejercicio, egresados, principalmente de la Licenciatura en Español y Literatura. Se espera que los resultados reúnan la síntesis de las experiencias y reflexiones en un modelo de lingüística escolar aplicable al currículo de las licenciaturas relacionadas con la enseñanza de la lengua materna y replicable en las Facultades de Educación y en la política curricular de lenguaje del MEN.

\section{Universidad y enseñanza para enseñar lengua}

Pensar la universidad como organismo que aporta a la construcción social, y la formación universitaria como espacio de adquisición de conocimientos especializados que desembocan directamente en las prácticas cotidianas de los profesionales, obliga a centrar 
el análisis en la última etapa del proceso educativo, es decir, en la relación que existe entre la formación profesional que ha obtenido el estudiante, y su ejecución en el entorno laboral donde aplica realmente el conocimiento adquirido.

En este sentido, importa indagar acerca de la adecuación de los saberes con respecto al mercado laboral, así como la capacidad de los egresados para actuar de conformidad con las exigencias y requerimientos del entorno donde se desempeñan. Preocupaciones de este tipo, son abordadas desde el Observatorio Laboral de la Universidad Tecnológica de Pereira en donde se han realizado distintos estudios con los que se busca establecer la pertinencia de la formación de los egresados, al tiempo que se caracteriza tanto su perfil profesional como su situación ocupacional y económica resultados del ejercicio de su profesión y de la formación académica (Vargas, 2010); en este sentido será posible "reafirmar, reorientar y/o actualizar los programas académicos de modo que respondan a las necesidades del mercado laboral" (Gaviria, 2009).

A nivel macro, la UNESCO (1996) como organismo internacional, recoge los aportes realizados por diferentes actores educativos llamando la atención sobre la brecha que existe entre la universidad y el mercado de trabajo, allí señala varios aspectos relevantes. Entre otros, resalta la necesidad de evaluar la pertinencia y eficacia de los distintos programas académicos en función de lo que ha ocurrido con los egresados y su desempeño laboral, de modo que desde allí sea posible determinar la adecuación de su proceso educativo. Alude también a las transformaciones socioculturales que impactan el campo formativo en dos direcciones: una orientada hacia la necesidad de preparar ahora para el futuro, por lo que se requieren proyecciones y estudios que muestren las líneas de acción requeridas según la evolución social; la otra en el sentido de que la creación y oferta del saber pone especial énfasis en la práctica como fin último, siendo las instituciones de educación superior las encargadas de fomentar estos procesos. De esta manera, para la UNESCO (1998), el perfil de los graduados debe ser de personas que han adquirido los conocimientos avanzados, generales o especializados, la capacidad para aplicarlos en situaciones concretas y las competencias sociales y comunicativas para actuar eficazmente en el entorno globalizado.

Sin embargo, se ha encontrado que existe una hegemonía de la formación teórica en detrimento de la formación práctica bajo el dominio de una tradición academicista que afecta también a los programas de formación docente, objeto del presente estudio; así, según Negrin (2003), frecuentemente el currículo universitario está construido con base en una relación entre teoría y práctica desde donde el ejercicio profesional es el resultado del traspaso de investigaciones teóricas, siendo las prácticas de enseñanza de los egresados docentes donde se evidencia la brecha entre los conocimientos impartidos en la universidad y el conjunto de saberes que demanda la profesión docente, específicamente 
la didáctica como puesta en marcha de estrategias orientadas a lograr el aprendizaje de los estudiantes en la educación básica.

Continuando con Negrin, tales estrategias requieren del docente el abordaje de cuestiones de orden epistemológico, psicológico y de relevancia social que le permitan actuar como mediador entre el saber sabio y el saber a enseñar descrito por Chevallard (1991). Es así como, el docente se enfrenta a una serie de situaciones, muchas de ellas propias del mundo contemporáneo que debe sortear a partir de los conocimientos y destrezas adquiridas durante su formación pregradual.

Particularmente, para el egresado, docente de lengua materna, estas situaciones inician en las necesidades particulares de los estudiantes a nivel del dominio del código oral y escrito que se particulariza en una sociedad donde los conceptos de leer y escribir han cobrado un significado distinto al que tenían hasta hace algunas décadas donde era suficiente reconocer las letras para considerarse alfabetizado, hasta llegar a un entorno mass mediático y tecnificado que obliga a desarrollar en los estudiantes habilidades de pensamiento inferencial que les permitan desenvolverse adecuadamente en el mundo cambiante y competitivo resultado de la globalización; esto lo reafirma el Ministerio de Educación Nacional (1998) cuando plantea dentro de las directrices para la enseñanza de la lengua materna, la importancia de formar al estudiante en la crítica y reflexión de la realidad desde donde sus competencias comunicativas le permitan enfrentar las exigencias del entorno.

Negrin (2003) se pregunta por las herramientas que utilizan los docentes para didactizar el conocimiento, encontrando en los libros de texto el apoyo más contundente pues allí se encuentra desde la planeación curricular hasta los mecanismos de evaluación, de tal manera, se hace imprescindible continuar con investigaciones como las realizadas por Olave y Rojas (2010) en torno a los ejercicios propuestos en los manuales escolares para la enseñanza de la gramática, de manera que, no sólo se aporte a nivel de trabajo con docentes en formación sino también se generen herramientas de apoyo para los maestros en ejercicio, al tiempo que se profundiza en el estudio, no solo a nivel gramatical sino en todos los niveles de estudio de la lengua.

Adicionalmente, Negrin explica que la adhesión de muchos docentes e instituciones educativas a los libros de texto, se presenta también por la dificultad que tienen de relacionar saberes provenientes de distintos campos que pongan la enseñanza de la lengua materna en línea transversal e interdisciplinaria con otras asignaturas, así, en trabajos realizados a nivel de educación superior (Cisneros, 2001, 2005, 2006, 2008, 2010, 2011; Cisneros, Olave y Rojas, 2010), se concluye la necesidad de que los docentes de lengua 
trabajen de manera cooperativa con los profesionales en otras áreas del conocimiento que permitan a los estudiantes aprender a leer y escribir desde cada una de las disciplinas, pues garantizar el desempeño adecuado en el uso de la lengua no es tarea exclusiva del docente de lenguaje.

Del mismo modo, los docentes necesitan integrar saberes culturales, escolares y cotidianos que respondan a los distintos contextos comunicativos. Es así como aparece nuevamente la necesidad de trascender el saber sabio de la linguística teórica, para integrarlo con los procesos reales de comunicación y construcción de pensamiento que permite el lenguaje.

Por todo lo anterior y según el consenso general ${ }^{1}$ sobre la importancia de la formación docente para enfrentar los retos del mundo contemporáneo, se hace evidente la necesidad de revisar las prácticas educativas actuales en la formación docente para determinar su repercusión en los procesos de enseñanza-aprendizaje al interior de las aulas de clase de las instituciones educativas, de modo que sea posible validar aquellas prácticas que responden a los objetivos planteados y dar nuevos enfoques a aquellas que dificultan los aprendizajes.

Retomando los postulados de la UNESCO (1998), es posible formar un docente de lengua que posea los conocimientos científicos requeridos, la capacidad para aplicarlos en el aula de clase desde el nivel inicial hasta la educación media, y poseer competencias sociales y comunicativas que le permitan orientar a sus futuros estudiantes de manera adecuada por el mundo del lenguaje. Todo lo anterior a través del mejoramiento continuo de los programas que preparan a los licenciados generando competencias laborales y profesionales como indicadores de calidad educativa (Cisneros \& Vega, 2011) universitaria.

Si los docentes forman parte activa en el proceso de transposición didáctica, es necesario reflexionar acerca de cuál es el estado de la formación de docentes de lengua materna en las licenciaturas. La preparación para el ejercicio pedagógico pretende resolver situaciones reales en entornos particulares que generalmente no corresponden a los modelos preconcebidos sino que requieren de una reformulación de las ideas en relación con las características particulares de dichas situaciones, en este sentido, la formación docente ha de centrarse en la reflexión crítica entre los problemas y las soluciones como

1 En reunión del mes de marzo en las instalaciones de la Universidad de Cartagena, de funcionarios del Ministerio de Educación Nacional con docentes de lengua, bibliotecarios y coordinadores de distintas instituciones educativas para revisar el Plan de Acción para el año 2012, se concluyó de manera general que los bajos resultados en materia de lectura y escritura de los estudiantes se deben principalmente a la falta de preparación docente para orientar de manera adecuada las prácticas en el aula. Así mismo, la UNESCO presenta la educación como el medio para construir una sociedad más justa y con mejor calidad de vida. (N. de los A.) 
estudio permanente y práctica adaptable, en lugar de modelos rígidos que impida su aplicación versátil. Así pues, en las licenciaturas debe existir una relación directa entre el campo del saber y su metodología de enseñanza según criterios de transposición teórico y práctica de orientación comunicativa.

Respondiendo entonces al desafío de relacionar el saber construido desde la teoría y adquirido en la universidad al saber construido en la práctica y requerido por la profesión docente, se busca responder a la pregunta: ¿Cómo realizar transposiciones didácticas de la lingüística teórica a una lingüística escolar?

Finalmente, consideramos que esta investigación se relaciona directamente con investigaciones a nivel internacional realizadas por autores como Emilia Ferreiro y Ana Teberosky en el nivel inicial, y Carlos Lomas, Ana Camps o Daniel Cassany, entre otros, a nivel de la educación básica. También, por las exigencias epistemológicas de la investigación, se hace necesario un marco teórico interdisciplinar concentrado en los avances teóricos y empíricos de la didáctica lingüística con enfoque psicolingüístico (Ferreiro y Teberosky, 1979), metacognitivo (Camps, 1997, 2006), constructivista social (Bronckart, 1985; Tolchinsky, 1993), comunicativo crítico (Cassany, 1990; Lomas y Tusón, 2009;) y sociodiscursivo (Parodi, 2005). Estos enfoques se transversalizan con el concepto de Transposición didáctica (Chevallard, 1991) y la construcción del concepto de Lingüística Escolar como producto intersubjetivo de los procesos dialógicos interdisciplinares.

\section{Sobre el estado de la cuestión}

Siguiendo a Gadamer (1977), la experiencia humana está estructurada linguísticamente, hablar y pensar, palabra y cosa son indisolubles, "el mundo es mundo en cuanto se expresa en el lenguaje, y el lenguaje existe en cuanto en él se representa el mundo"; este postulado se aleja de comprender el lenguaje como un sistema de signos que designan un mundo prelingüísticamente conocido o un instrumento al servicio del hombre; por el contrario, el lenguaje es concebido como medio de interpretación y conocimiento de la realidad, afirmando que la experiencia del mundo se produce paralelamente al lenguaje. En este sentido, cobra especial importancia la forma como se aborda la enseñanza del lenguaje en la escuela, las perspectivas utilizadas y las posibilidades que ofrece han de responder, no tanto al conocimiento conceptual de las normas lingüísticas, sino al poder interpretativo que se suscita a partir del dominio del lenguaje.

Los estudios que se han preocupado por la educación lingüística en la escuela desde el nivel inicial hasta el secundario se inscriben en el campo de la lingüística aplicada, siendo coherentes con los avances teóricos en la disciplina que se han suscitado en el transcurso del tiempo, procurando articularlos permanentemente con los saberes 
didácticos. En el estado del arte actual, a nivel de la educación primaria, las investigaciones de Emilia Ferreiro y Ana Teberosky (1979) en Argentina, con base en los estudios de Piaget, lograron ahondar en el campo de la psicogénesis de la lengua escrita para comprender la forma como los niños en edad preescolar aprenden el sistema de escritura, y a partir de allí, formular las bases teóricas sobre las cuales han de fundamentarse los procesos de enseñanza-aprendizaje. Ferreiro entiende el desarrollo de la lengua escrita en el niño, no como un proceso de aprendizaje memorístico de correspondencia fonográfica, sino como la travesía por diferentes etapas que le permiten ir haciendo hipótesis y comprobándolas acerca de cómo funciona el sistema. En esta misma línea, Tolchinsky (1993) llama la atención sobre los principios constructivistas que sirvieron de base para el estudio de la psicogénesis de la lengua: en primer lugar, la visión del niño como sujeto activo en el proceso de aprendizaje, que al estar inmerso en una cultura letrada, se pregunta por la lengua escrita aún antes de ingresar al sistema escolar; el segundo, al que responde el trabajo de Ferreiro, se relaciona con el estudio de la forma como los niños aprenden el lenguaje escrito, finalmente, la importancia de que el sujeto reconstruya el conocimiento, sacando por sí mismo conclusiones sobre el funcionamiento del sistema.

Una vez adquirido el dominio del código escrito, la enseñanza de la lengua continúa en el nivel secundario donde los autores (Lomas y Tusón, 2009; Camps, 1997; Cassany, 1990) coinciden acerca del enfoque comunicativo que debe imperar en los procesos de enseñanza-aprendizaje de la lengua materna, desde allí, los objetivos pedagógicos trascienden el conocimiento enciclopédico de la gramática, para orientarlo hacia la comunicación efectiva, el actuar en el mundo desde la comprensión y producción de distintos tipos de texto, así como el desarrollo del pensamiento crítico y reflexivo que les permita tomar postura frente a los diferentes fenómenos de la realidad contemporánea.

Para Ferreiro, estos conceptos se encuentran inmersos en la idea de escritura como sistema de representación; para Lomas y Tusón, significa lo que ellos denominan emancipación comunicativa, que integra todos los niveles de la lengua y todas las ciencias presentes en el estudio del lenguaje, en un intento por articular aspectos que, según los autores, casi siempre han estado demasiado alejados en el ámbito de la investigación linguística y educativa (teoría y práctica, investigación y acción, saber y saber hacer) y preparan el camino para evitar las inercias pedagógicas que se presentan en el aula al entender la lengua como un objeto de estudio.

En el mismo sentido, Camps (1993) subraya el papel de las escuelas discursivas que pretenden realizar avances hacia una sociocrítica de la comunicación a partir de estudios explicativos y, en mayor o menor medida, propositivos centrados en la trascendencia social de los usos de la lengua, esta orientación es especialmente interdisciplinar y tiene como 
objetivo ir más allá de las opciones sintagmáticas y sintácticas, lo cual se traduce, por un lado, en tomar en cuenta aspectos del contexto, y por el otro (y en consecuencia), por una aproximación funcional de la organización de la lengua poniendo en cuestión la unidad frase y poniéndole las nociones de enunciado, de texto o de discurso como formas de la actividad humana'. Esta orientación general, que, a pesar de la heterogeneidad de enfoques es la que se encuentra en la base de muchas de las nuevas propuestas de enseñanza de la lengua".

Parodi (2005) presta especial atención al estudio de la lengua en relación directa con las profesiones, es decir, los procesos lingüísticos particulares que sostienen el conocimiento especializado y hacen de la lengua una herramienta transversal al entenderla como recurso vehicular básico para la enseñanza y transmisión de todo tipo de ideas y de contenidos que integran cualquier currículum escolar.

Mientras que Parodi realiza un estudio interdisciplinar de la linguística como herramienta transversal entre las disciplinas que brinda acceso al conocimiento, Bronckart (1985) analiza los componentes internos de la lingǘstica que intervienen en estos procesos comunicativos, así, sostiene que los estudios lingüísticos como la pragmática, la teoría de la enunciación o la gramática textual, "tienen como objetivo ir más allá de las opciones sintagmáticas y sintácticas", esto significa tomar en cuenta los aspectos del contexto y, por lo tanto, entender la lengua bajo un enfoque funcional. Sin embargo, siguiendo a Camps (1993) las diversas orientaciones de los estudios lingüísticos dificultan su relación con los procesos de enseñanza que tampoco se corresponden con la formación de docentes en lengua egresados de universidades donde se imparte el enfoque estructuralista.

En Colombia no se han desconocido estos avances y se han hecho esfuerzos por promover la investigación en la didáctica linguística, a través de las redes de lenguaje que funcionan en las diferentes regiones del país y buscan la discusión y actualización de las prácticas de lenguaje al interior de las instituciones educativas articulando todos los niveles de formación y resaltando una pedagogía por proyectos (Varios, 2005). Así mismo, la reforma curricular que permitió avanzar en una política curricular con enfoque semántico-comunicativo que se evidencia en los Lineamientos Curriculares de Lengua Castellana propuestos por el Ministerio de Educación Nacional (1998) y en el que se fundamentan, a nivel general, las prácticas educativas en las instituciones. Sin embargo, además de estos documentos, es difícil encontrar estudios que ahonden en la enseñanza de la lengua materna que abarquen, no sólo el tema de la lectura y la escritura sino una didáctica de tipo más global que incluya la lingüística como objeto de saber en la escuela, base para el desarrollo de la competencia comunicativa. 
Son importantes los aportes de la Red de Transformación para la formación docente en lenguaje, no obstante, están centrados en el problema de la lectura y la escritura y no en el asunto lingüístico. En el desarrollo del presente proyecto ahondaremos en las publicaciones de esta red con el fin de aprovechar al máximo sus hallazgos. En materia de enseñanza de la lengua hay también algunos documentos que se refieren a la enseñanza de la lengua extranjera y enfocados a las comunidades indígenas, al respecto veremos qué tan pertinentes sean para nuestra investigación.

En Garzón, Grajales y Guzman (2010) se estudia la naturaleza de los saberes enseñados en el curso Construcción y Didáctica de la Lengua Materna dentro del programa de Licenciatura en Pedagogía Infantil de la Universidad Tecnológica de Pereira, allí los autores parten de la importancia de conocer la naturaleza de los saberes y su forma de transmisión, poniendo en juego el rol del docente y su relación con dicho saber, es esta relación la que permite determinar el discurso, los recursos y las intencionalidades para hacer enseñable el curso de construcción y didáctica de la lengua materna.

\section{Sobre algunos conceptos}

Hablar de enseñanza de la lengua implica también tener claridad sobre el concepto de transposición didáctica trabajado por Chevallard (1991) y definido como "un contenido del saber sabio que haya sido designado como saber a enseñar sufre a partir de entonces un conjunto de transformaciones adaptativas que van a hacerlo apto para tomar lugar entre los objetos de enseñanza". Desde esta perspectiva se hace posible analizar el proceso de adaptación por el cual la linguística teórica se convierte en un conocimiento para ser enseñado en la escuela básica; en esta transición se observa la existencia de un abismo entre ciencia y educación, donde el desarrollo teórico de la lingüística no ha encontrado su aplicación en la linguística escolar en torno a los temas y metodologías que deben implementarse al interior del aula para hacer de ella un conocimiento significativo; el saber científico se transfiere directamente al saber enseñado en la escuela realizando solo una simplificación del conocimiento, mas no una adecuación de los contenidos a partir de las necesidades comunicativas del estudiante (Olave y Rojas, 2011).

Bronckart (1995) alude a la transposición didáctica de la lengua materna que debe fundamentarse bajo tres aspectos básicos, el primero de los cuales permite crear un aparato conceptual que fije los conceptos de manera adaptada para alumnos y profesores, cabe resaltar que se utiliza el término adaptación y no simplificación, pues los contenidos de la linguística habrán de responder a las necesidades del entorno escolar con enfoque comunicativo y funcional; posteriormente, será necesario identificar las dificultades que poseen los alumnos para derivar de allí una lista estructurada de objetivos de enseñanza, 
finalmente será posible elaborar las correspondientes secuencias didácticas. Como ya se ha expresado anteriormente, la presente investigación busca trabajar sobre esta primera etapa del proceso de transposición didáctica propuesto por Bronckart.

De otro lado, se entiende por lingüística teórica (Tusón, 2002) aquella que busca construir modelos que definan las estructuras de la lengua, una disciplina que dicta postulados para explicar los fenómenos lingüísticos desde la conceptualización, presentando la lengua como algo unitario, sistemático y homogéneo. En el campo de la enseñanza aparece la lingüística aplicada, la pedagogía de la lengua y la didáctica lingüística. La primera se refiere a una rama de la lingüística que utiliza los conocimientos de la linguística teórica para mejorar las condiciones de la comunicación verbal. La pedagogía de la lengua abarca un campo de acción más amplio en donde es posible reflexionar acerca de la enseñanza del lenguaje a nivel psicosocial que orienta las directrices bajo las cuales se formularán las metodologías de enseñanza; para la elaboración del presente estudio será necesario analizar el tipo de pedagogía que orienta una linguística escolar. Por su parte, la didáctica de la lengua es definida por Camps (1993) como "una disciplina que tiene su origen en la práctica y en ella se sitúa también su objetivo", por tal razón, cobra especial importancia el análisis de las interacciones reales que responden a las expectativas implícitas y explícitas sobre los conocimientos lingüísticos que se esperan de cualquier miembro de la sociedad; señala además que tal didáctica de la lengua abre un espacio para la interacción entre las prácticas de aula y los procesos de enseñanzaaprendizaje de una materia específica que en este caso es la lengua.

Dentro de una línea más amplia de la didáctica, el concepto de profesional reflexivo acuñado por Schön (1992) plantea que el éxito del docente depende en buena parte de su habilidad para manejar la complejidad y resolver problemas prácticos del aula escolar, es decir, el saber teórico o académico pasa a ser considerado instrumento en los procesos de reflexión que se producen cuando la teoría se integra de forma significativa al quehacer diario; para el caso que aquí se postula, es la lingüística escolar donde convergen ambos elementos (teoría y práctica) para responder a los requerimientos del contexto y al dominio efectivo en el uso del lenguaje.

En el mismo sentido, Quintar (2002) resalta la importancia de enseñar para la autonomía del sujeto centrando los procesos de aula en torno a la reflexión colectiva y la validez de los constructos teóricos producidos por los docentes para resignificar las prácticas, al igual que Zambrano (2002) hace especial énfasis en el concepto de didáctica como técnica que da lugar a la práctica pedagógica relacionada directamente con la reflexión del quehacer. Tal reflexión responde a la necesidad de problematizar el conocimiento como una actividad que relaciona directamente la crítica de la realidad, 
objeto de estudio y la autocrítica del sujeto que se apropia de esa realidad (Zemelman, 1992:80). Lo anterior es de especial relevancia para la enseñanza de la lengua en tanto que resalta la necesidad de orientar las prácticas pedagógicas, no hacia el dominio conceptual del conocimiento, sino al desarrollo de la capacidad crítica dada a partir del uso eficiente del lenguaje.

\section{Metodología}

Proponer estrategias de transposición didáctica de la linguiística teórica a una linguística escolar, para integrarlas a los currículos de didáctica del lenguaje implica, en primera instancia, realizar una revisión documental que garantice el conocimiento y comprensión del estado actual de la enseñanza de la lengua y su didáctica como asignatura a nivel de educación superior; esta revisión se realizará a nivel diacrónico y sincrónico a través de una hermenéutica documental tanto de los currículos de didáctica de la lengua como de la lingǘrstica en tanto ciencia de nivel teórico.

Una vez descritos y analizados en profundidad tales currículos a la luz de las teorías antes expuestas, se procederá a realizar entrevistas narrativas a una muestra de docentes en ejercicio egresados de licenciaturas relacionadas con la enseñanza de la lengua materna, periodo en el cual se cuenta con profesionales en educación que poseen una experiencia suficiente en este campo de enseñanza; de esta manera se hará visible el impacto de la formación universitaria en las prácticas reales dentro del aula de clase a nivel del ejercicio del maestro, en su doble dominio de la lengua y la didáctica.

A partir de estos resultados, se entrará en una segunda fase de investigación donde se diseñarán estrategias para "enseñar a enseñar" la lingüística escolar, estrategias que parten de las dificultades encontradas por los docentes en el aula y la forma como se hace posible direccionar los procesos didácticos para enfrentar los desafíos de la educación actual. Se trata de dar respuesta a los contenidos y métodos de enseñanza que corresponden al desarrollo de la competencia comunicativa del estudiante de educación básica en todos los niveles y transversalizado por la ciencia lingüística y sus disciplinas particulares; así mismo, a partir de las teorías lingüísticas y la orientación pedagógica.

Los resultados de la segunda fase, bajo el modelo de orientaciones estratégicas para la formación de docentes en lenguaje, serán sometidos a juicio de expertos nacionales e internacionales a través del método Delphi, reformulados con base en esta revisión y divulgados en el medio académico, como aporte pedagógico concreto para las aulas universitarias de licenciaturas relacionadas con la enseñanza de la lengua materna. 
En la medida en que los programas de formación de formadores incluyan dentro de sus dinámicas de aula el espacio para la participación activa de los estudiantes haciéndolos gestores de su propio aprendizaje, será posible, no sólo que los conocimientos se construyan de forma crítica desde la comprensión de su origen, sino también que trasciendan a las aulas de educación básica como rasgo metodológico acorde con los requerimientos educativos de la sociedad contemporánea.

\section{A manera de cierre}

A pesar de los numerosos avances en las orientaciones sobre la enseñanza de la lengua materna, aún queda un escollo que dificulta el trabajo en el aula en tanto que el énfasis que se ha puesto hasta ahora ha sido a nivel de la didáctica más que en relación con la lingüística como ciencia que requiere de una rama especializada en su forma de transmisión en la escuela; esta afirmación se sustenta en el trabajo de Olave y Rojas (2011) donde se afirma que se tiene claridad acerca del enfoque comunicativo en la enseñanza de la lengua al interior de la interacción social, sin embargo, no hay claridad acerca del papel específico de la gramática [y la lingüística en general] para el trabajo en el aula.

La profesionalización docente en las licenciaturas incluye la revisión del paso de la lingüística teórica a la escolar. En la dinámica de esta transición se encuentra el núcleo de la investigación propuesta: la construcción de un diálogo interdisciplinar entre lingüistas y pedagogos que se integre al currículo de didáctica de la lengua materna para avanzar en la profesionalización de licenciados más propositivos en las aulas de clase.

El desafío en el actual estado del arte es, entonces, elaborar respuestas a las cuestiones surgidas de la interacción entre los requerimientos de la sociedad y las propuestas didácticas de enseñanza de la lengua materna, de modo que se establezca un feedback entre carencias y nuevos conocimientos, entre innovaciones y valoraciones de la teoría y la práctica según las pautas de una investigación rigurosa con base en situaciones reales.

\section{Referencias bibliográficas}

Bronckart, J. P. (1985). Las ciencias del lenguaje, un desafío para la enseñanza. París: Unesco.

Camps, A. (1993). Didáctica de la lengua: la emergencia de un campo científico específico. Infancia y aprendizaje, 62/63. Universidad Autónoma de Barcelona. Recuperado de http://www. educacion.objectis.net/primer-ciclo/documentos-dereferencia/objeto $\% 20 \mathrm{de} \% 201 \mathrm{la} \% 20$ didactica\%20camps.doc 
Camps, A. (1997). La enseñanza y el aprendizaje de la composición escrita. Signos. Teoría y práctica de la educación, 20, 24-33.

Camps, A., y Zayas, F. (coords). (2006). Secuencias didácticas para aprender gramática. Barcelona: Grao.

Cassany, D. (1990). Enfoques didácticos para la enseñanza de la expresión escrita. Comunicación, lenguaje y educación, 6, 63-80. Madrid: Universitat Pompeu Fabra. Disponible en: http:// www.upf.edu/pdi/dtf/daniel_cassany/enfoques.htm

Cisneros-Estupiñán, M., y Vega, V. (2011). En busca de la calidad educativa a partir de los procesos de lectura y escritura. Pereira: Universidad Tecnológica de Pereira.

Cisneros-Estupiñán, M., Jiménez, H., y Rojas, G. (2010). “Alfabetización académica y profesional como directrices de la acción formativa en la Educación Superior”. En: Giovanni Parodi (editor) (2010). Alfabetización Académica y Profesional: Leer y escribir desde las disciplinas. Santiago de Chile: Academia Chilena de la Lengua y Editorial Planeta.

Cisneros-Estupiñán, M. (2008). "Lectura y escritura en el momento de ingreso a la educación superior". En: Varios autores (2008), Los desafios de la lectura y la escritura en la educación superior: caminos posibles. Cali: Universidad Autónoma de Occidente.

Cisneros-Estupiñán, M. (2006). Lectura y escritura en la universidad: una investigación diagnóstica. Pereira: Universidad Tecnológica de Pereira.

Cisneros-Estupiñán, M. (2005) . "Mejorar los procesos lectoescriturales desde la educación básica", En: Varios autores (2005). Didáctica de la lengua materna: estado de la discusión. Cali: Universidad del Valle, ICFES.

Cisneros-Estupiñán, M. (2001). Hacia la enseñanza de la lengua materna en el nivel universitario. Interlenguajes, 2(1), 67-81.

Cisneros-Estupiñán, M., Olave, G., y Rojas, I. (2010). La Inferencia en la Comprensión Lectora: de la Teoría a la Práctica en la Educación Superior. Pereira: Universidad Tecnológica de Pereira.

Chevallard, Y. (1991). La transposición didáctica: Del saber sabio al saber enseñado. Argentina: Aique.

Ferreiro, E., y Teberosky, A. (1979). Los sistemas de escritura en el niño. España: Siglo XXI.

Gadamer, H. G. (1977). Verdady método. Salamanca: Editorial Sígueme.

Garzón, C., Grajales, G., y Guzman, D. (2010). La enseñanza dellenguaje como saber universitario: estudio epistemológico, didáctico, textual del curso "construcción y didáctica de la lengua materna”. Disponible en: http://hdl.handle.net/123456789/1439 
Gaviria, J. y otros (2009). Evaluación de la pertinencia de la formación de pregrado y su articulación con el campo laboral del programa de Licenciatura en Música de la Universidad Tecnológica de Pereira. Disponible en: http://repositorio.utp.edu.co/xml/ handle/123456789/1636

Lomas, C., y Tusón, A. (2009). Enseñanza del lenguaje, emancipación comunicativa y educación crítica: el aprendizaje de competencias comunicativas en el aula. México: Edere.

Ministerio de Educación Nacional de Colombia (1998). Lineamientos curriculares de lengua castellana. Bogotá: Magisterio.

Negrin, M. (2003). Saberes teóricos y práctica docente: Razones de un desencuentro. Educación, Lenguaje y Sociedad, 1(1), 307-312.

Olave, G., y Rojas, I. (2010). Análisis crítico de ejercicios usados para enseñar gramática en los manuales escolares de la educación básica secundaria. Tesis de Maestría. Pereira: Universidad Tecnológica de Pereira.

Olave, G. y Rojas, I. (2011). La Gramática al tablero. Pereira: Universidad Tecnológica de Pereira.

Parodi, G. (2005). Comprensión de textos escritos. Buenos Aires: Eudeba.

Quintar, S. (2002). La enseñanza como puente a la vida. Argentina: Universidad Nacional del Comahue.

Schön, D. (1992). El profesional reflexivo. Barcelona: Paidós.

Tolchinsky, L. (1993). Aprendizaje del lenguaje escrito. Barcelona: Anthropos.

Tusón, A. (2002). Iguales ante la lengua, desiguales en el uso. En: Lomas, C. El aprendizaje de la comunicación en las aulas. Barcelona: Paidós.

UNESCO. (1998). ¿Están los universitarios listos para el campo de trabajo?. Disponible en: http://www.iesalc.unesco.org.ve/index.php?option=com_content\&view=article\&id=2696 \%3Aiestan-los-universitarios-listos-para-el-mercado-de-trabajo\&catid=126\%3Anoticiaspagina-nueva\&Itemid $=712 \&$ lang $=\mathrm{es}$

Vargas, E., y otros. (2010). Evaluación de la pertinencia de programas de pregrado en la Universidad Tecnológica de Pereira. Pereira: Universidad Tecnológica de Pereira.

Varios autores (2005). Didáctica de la lengua materna: estado de la discusión. Cali: Universidad del Valle, ICFES.

Zambrano, A. (2002). Los hilos de la palabra. Cali: Nueva Biblioteca Pedagógica.

Zemelman, H. (1992). Horizontes de la razón. Barcelona: Anthropos. 